\title{
Anti-inflammatory coating of hernia repair meshes: a 5-rabbit study
}

\author{
M. Bredikhin ${ }^{1}$ - D. Gil ${ }^{1}$ J. Rex ${ }^{1} \cdot$ W. Cobb $^{2} \cdot$ V. Reukov ${ }^{1} \cdot$ A. Vertegel $^{1}$ (]) \\ Received: 21 August 2019 / Accepted: 4 January 2020 / Published online: 5 February 2020 \\ (c) Springer-Verlag France SAS, part of Springer Nature 2020
}

\begin{abstract}
Purpose Polymeric mesh implantation has become the golden standard in hernia repair, which nowadays is one of the most frequently performed surgeries in the world. However, many biocompatibility issues remain to be a concern for hernioplasty, with chronic pain being the most notable post-operative complication. Oxidative stress appears to be a major factor in the development of those complications. Lack of material inertness in vivo and oxidative environment formed by inflammatory cells result in both mesh deterioration and slowed healing process. In a pilot in vivo study, we prepared and characterized polypropylene hernia meshes with vitamin E ( $\alpha$-tocopherol)-a potent antioxidant. The results of that study supported the use of vitamin $\mathrm{E}$ as potential coating to alleviate post-surgical inflammation, but the pilot nature of the study yielded limited statistical data. The purpose of this study was to verify the observed trend of the pilot study statistically.

Methods In this work, we conducted a 5-animal experiment where we have implanted vitamin E-coated and uncoated control meshes into the abdominal walls of rabbits. Histology of the mesh-adjacent tissues and electron microscopy of the explanted mesh surface were conducted to characterize host tissue response to the implanted meshes.

Results As expected, modified meshes exhibited reduced foreign body reaction, as evidenced by histological scores for fatty infiltrates, macrophages, neovascularization, and collagen organization, as well as by the surface deterioration of the meshes. Conclusion In conclusion, results indicate that vitamin E coating reduces inflammatory response following hernioplasty and protects mesh material from oxidative deterioration.
\end{abstract}

Keywords Hernia $\cdot$ Vitamin E $\cdot$ Polypropylene mesh $\cdot$ Rabbit model

\section{Introduction}

An abdominal wall hernia is a protrusion of tissues through the musculoaponeurotic wall of the abdomen. This type of hernia is very common, with an estimated 800,000 new cases in the United States and 20 million repair procedures worldwide every year $[1,2]$. While many of these are attributed to $10-30 \%$ patients following laparotomy, the overall incidence rate is likely to increase as obesity continues to rise in the US [2].

The use of polymeric meshes for the reinforcement of hernia repairs was first pioneered in 1958 by Usher et al. [3]. Mesh implantation has become heavily practiced, as these

A. Vertegel

vertege@clemson.edu

1 Department of Bioengineering, Clemson University, 301 Rhodes Hall, Clemson, SC 29634, USA

2 Department of Surgery, The Hernia Center, Prisma Health, 2104 Woodruff rd., Greenville, SC 29607, USA implants were shown to strengthen the rupture site and produce less than half the recurrence rate of the sutured closures [4]. Most of the meshes used nowadays are knitted from monofilament polypropylene. Polypropylene is affordable, chemically inert, and able to withstand sterilization while retaining flexibility [5]. Porosity of the mesh suggests the enhanced tissue integration and the reduced foreign body reaction. However, many biocompatibility issues such as mechanical failure, seroma formation, and infection remain to be a concern for hernia repair [6].

Chief among post-hernioplasty complications is chronic pain, which has been reported in nearly $30 \%$ of patients [7]. While the exact cause of the chronic pain has not been established yet, lack of material inertness in vivo and oxidative environment induced by inflammatory cells in the incision area appear to be the key factors in poor mesh performance $[2,5,8]$. White blood cells are recruited at the interface between tissue and mesh fibers within minutes to hours after the implantation. Recognition of a non-host surface by these cells induces an exocytotic release of lytic enzymes 
and oxidants to degrade the foreign body. These oxidants are mainly reactive oxygen species (ROS), with superoxide anion, peroxynitrite, and hydroxyl radical being among the most notable ones. These compounds have been shown to both deteriorate the surface of the mesh and penetrate the fibers, therefore altering both bulk and surface properties [5, 9]. Hypochlorite ions produced by myeloperoxidase found in neutrophils and macrophages have also been reported to assist in oxidative degradation of the implant [10].

Different strategies have been proposed to address various biocompatibility concerns associated with the hernia repair meshes. These include alterations in a pore size and shape, transition from a heavyweight to a lightweight mesh, knitting design of the fibers, etc. Levinson et al. showed that modifying the knits in the mesh significantly improves device mechanical performance and enhances tension-free repair [11]. Lightweight mesh has been reported to induce fewer peritoneal adhesions than a heavyweight mesh does [12]. Other studies showed that the meshes with larger pores and complex pore shape promote better tissue ingrowth than their counterparts with smaller circular/oval pores [13]. For example, Deeken et al. demonstrated that the meshes with hexagonal pores perform significantly better in terms of host tissue response than the meshes with diamond-shape or circular pores [13]. Nevertheless, these strategies only yield incremental improvements in the overall long-term post-operative complications.

Lately surface coatings of implants have gained an increased attention. Modifying the surface may be advantageous in two generic ways: protection of the implant from degradation and mitigation of the foreign body reaction. Thus, hernia mesh had been subjected to various coatings in many studies [14-16]. Emans et al. proposed that coating hernia mesh with oxygenated cellulose hydrogel reduces tissue adhesions [14]. Other researchers focused on preventing clinical infections associated with hernioplasty. For example, coating meshes with antimicrobial enzyme proteases, such as lysostaphin, has been proven to significantly reduce the infection rate and bacterial colonization of the mesh [17]. In an attempt to mitigate inflammatory response after implantation, nitric oxide was adsorbed to the surface of mesh with polyvinyl alcohol used as a scaffolding carrier [15]. The study revealed reduced inflammatory response to these meshes when compared to uncoated meshes but, unfortunately, exogenous NO has proved to be cytotoxic [15]. Therefore, the search for an optimal anti-inflammatory coating of hernia repair meshes is still in progress.

Vitamin E refers to a class of compounds that include tocopherols and tocotrienols, with $\alpha$-tocopherol being the most common one. In human body, vitamin $\mathrm{E}$ acts as a fatsoluble antioxidant by scavenging free radicals and preventing associated damage to the tissues. It showed great efficacy in vivo for the conditions where oxidative stress plays a crucial role. For example, infusion of vitamin $\mathrm{E}$ is used in ultra-high molecular weight polyethylene hip and knee implants to make them resistant to oxidation [18]. Regarding hernia repair, intraperitoneal injections of vitamin $\mathrm{E}$ following mesh implantation have been found to reduce tissue adhesions in a rat model [19]. Finally, vitamin E is generally recognized as safe (GRAS) by FDA, which reduces safety concerns and is expected to facilitate regulatory approval of vitamin E-coated meshes.

In a pilot one-rabbit study, we showed that vitamin E-coated meshes exhibited reduced foreign body response and improved wound healing [20]. Furthermore, in vitro release of vitamin $\mathrm{E}$ from the coated mesh was desirably slow, with $70 \%$ of the coating remaining after 10 days of exposure to albumin solution, suggesting sustained release and long-term protection [20]. However, pilot nature of the study yielded limited statistical data.

In this work, we have conducted a 5-animal experiment where we have implanted vitamin E-coated and uncoated control meshes into the abdominal walls of rabbits utilizing a retromuscular approach to hernia repair. In addition to the in-depth histological evaluation, we have also conducted scanning electron microscopy studies to characterize the explanted mesh surface. We hypothesized that our results would be in a close agreement with those of the pilot study: coating hernia meshes with vitamin $\mathrm{E}$ would mitigate inflammatory response around the implant and lower the extent of surface deterioration of the mesh. Testing 5 animals can statistically confirm the in vivo trend that we have observed before.

\section{Materials and methods}

\section{Materials}

Prolene ${ }^{\circledR}$ polypropylene mesh (Ethicon Inc., a Johnson \& Johnson company, Langhorne, PA, USA) was kindly donated by the Carolinas Medical Center (Charlotte, NC, USA). It is a heavyweight non-absorbable mesh with a density of $80 \mathrm{~g} /$ $\mathrm{m}^{2}$. Vitamin E ( $\alpha$-tocopherol), 95\% medical grade ethanol, and sodium hypochlorite solution (10-15\% available chlorine) were purchased from Sigma-Aldrich (Sigma-Aldrich, St. Louis, MO, USA).

\section{Preparation of vitamin E-coated meshes}

Coating and sterilization methods were the same as in the pilot study [20]. Briefly, vitamin E was dissolved in 95\% medical grade ethanol $(100 \mathrm{mg} / \mathrm{ml})$ and this solution was transferred into a 6-well plate containing pieces of surgical mesh ( $10 \mathrm{ml}$ per well). Samples were incubated overnight in the dark at $4{ }^{\circ} \mathrm{C}$. Meshes were then air-dried overnight in the 
dark at room temperature under sterile conditions. Uncoated control meshes were incubated in pure ethanol. For the animal studies, the size of all meshes was $3 \mathrm{~cm} \times 3 \mathrm{~cm}$. They were cut with sterile metal scissors and re-sterilized under UV irradiation for $60 \mathrm{~min}$ prior to implantation.

\section{Animal study}

Five 13-week old male New Zealand white rabbits were used in this study. This number was chosen based on a power analysis of the data collected from the pilot study. The average weight of the animals was approximately $3300 \mathrm{~g}$. All animal experimental procedures were conducted according to the protocol approved by the institutional animal care and use committee of Clemson University (AUP-2015-019). The rabbits were hosted in Godley-Snell Animal Facility at Clemson University and treated according to the established standards. Anesthesia of the animals prior to surgeries was done by injection of acepromazine $1 \mathrm{mg} / \mathrm{kg}$, ketamine HCL $33 \mathrm{mg} / \mathrm{kg}$, atropine $0.02-0.05 \mathrm{mg} / \mathrm{kg}$, and buprenorphine $0.05 \mathrm{mg} / \mathrm{kg}$.

Implantation surgery was performed using the same protocol as in the pilot study [20]. Following skin sterilization, a $5-\mathrm{cm}$ incision was cut in the midline of the animals' abdominal wall. The abdominal cavity was carefully penetrated along the linea alba. Laterally, the posterior fascia was incised to the rectus muscle. The posterior sheath was then carefully removed from the rectus muscle. At the semilunar line, the dissection transitioned to the plane between the transversus abdominis and internal oblique muscles laterally. An intermuscular plane was formed to accommodate a $3 \mathrm{~cm} \times 3 \mathrm{~cm}$ mesh. The posterior sheath was then re-approximated in the midline with 4-0 polydioxanone suture in a running fashion. One vitamin E-coated and one uncoated mesh were implanted into each rabbit on left and right sides, symmetrically with respect to the linea alba. As it was observed in the pilot study, coated meshes were not sticky and their handling was not different from the uncoated meshes. The mesh pieces were secured to the muscle inferiorly at the four corners with 4-0 polydioxanone suture. The anterior fascia was then closed in the midline with 4-0 polydioxanone suture in a running fashion. Skin was sutured with 3-0 polydioxanone sutures.

Five weeks after the surgery, all rabbits were euthanized, and tissue-mesh explants were excised in en bloc fashion. Explanted specimens contained fascia, mesh, abdominal muscles and skin and were placed in $10 \%$ formaldehyde solution for long-term storage. For each specimen, $2.5 \mathrm{~cm} \times 2.5 \mathrm{~cm}$ piece were used for histology studies and the remaining $0.5 \times 0.5 \mathrm{~cm}$ pieces were cut for the microscopical analysis.

\section{Histology studies}

Samples containing the mesh and all layers of tissue were cut from the specimens stored in $10 \%$ formalin and embedded in paraffin. $10 \mu \mathrm{m}$ sections were cut using a microtome. The samples were then stained with hematoxylin and eosin (H\&E) and Masson's trichrome, followed by observation under optical microscope. A modified histological scale used in this study was adapted from the literature [21] and ISO 10993-6 (see Table 1). Inflammatory response was assessed by counting the number of macrophages and foreign body giant cells (FBGCs) found in the vicinity of the meshes. Semi-quantitative characterization of collagen organization, neovascularization, and fatty infiltrates was performed and graded by an independent experienced histologist in a double-blinded manner.

\section{Microscopy studies}

Micromorphological characteristics of the explants were assessed using Scanning Electron microscopy (Hitachi S4800, Clemson, SC, USA). Prior to the analysis, tissue residues were removed according to the protocol adapted from previous studies [2]. Briefly, major tissue pieces were removed by forceps under sterile conditions. To completely remove residual tissue, the explants were incubated in sodium hypochlorite solution (10-15\% available chlorine) for $12 \mathrm{~h}$ at $50{ }^{\circ} \mathrm{C}$. No visible tissue on the mesh surface was observed after such treatment. Pristine mesh was also subjected to this procedure to show that it does not deteriorate the mesh. The meshes were then washed three times with an

Table 1 Histological scoring system

\begin{tabular}{|c|c|c|c|c|c|}
\hline \multirow[t]{2}{*}{ Response } & \multicolumn{5}{|c|}{ Score } \\
\hline & 0 & 1 & 2 & 3 & 4 \\
\hline Collagen organization & None & Minimal organization & Mild organization & Moderate organization & Well-organized \\
\hline Neovascularization & None & $\begin{array}{l}\text { Minimal capillary prolifera- } \\
\text { tion }\end{array}$ & Mild amount of capillaries & Moderate bands of capillaries & Abundant vascularity \\
\hline Fatty infiltrate & None & Minimal amount of fat & Mild amount of fat & $\begin{array}{l}\text { Broad/elongated fatty infil- } \\
\text { trates }\end{array}$ & Severe fatty infiltrates \\
\hline
\end{tabular}


excess of DI water. Prior to the SEM visualization, samples were sputter-coated with $5 \mathrm{~nm}$ layer of Pt.

\section{Statistical analysis}

Numerical data are presented as a mean and standard deviation. Student's $t$ test and one-way ANOVA were used for comparison between vitamin E-coated mesh data and uncoated mesh data. A $p$ value of less than 0.05 was assumed to indicate statistically significant difference.

\section{Results}

\section{Coating concentration}

The specific surface area of the meshes was determined by low-temperature nitrogen adsorption using Brunauer-Emmett-Teller (BET) analysis and was calculated to be $3.4 \mathrm{~m}^{2} / \mathrm{g}$ [20]. Since the maximum binding yield of vitamin $\mathrm{E}$ to mesh surface $\left(\sim 6 \mathrm{mg} / \mathrm{in}^{2}\right)$ was observed at the initial coating concentration of $100 \mathrm{mg} / \mathrm{ml}$ in the pilot study, this concentration was used in this study [20].

\section{Wound healing}

All rabbits survived until the sacrifice date. Wounds generally healed well with no evident dehiscence, infections, or other complications.

\section{Histological evaluation}

Haematoxylin eosin staining was done to evaluate the inflammatory response to plain and vitamin E-coated meshes (Fig. 1). As seen in Figs. 1 and 2, meshes are surrounded by macrophages. However, in the case of uncoated meshes the number of these cells is significantly larger $(p=0.015$, $n=30$, see Table 2 ). For all animals individually, the difference was statistically significant too. Additionally, little to no evidence of foreign body giant cells was found in the
Fig. 1 Microphotographs of representative haematoxylin and eosin-stained histological slides under $\times 200$ magnification. Asterisks designate the areas where mesh fibers were originally present. Yellow arrows indicate presence of fatty infiltrates. a, c Uncoated mesh. b, d Vitamin E-coated mesh (color figure online)
Fig. 2 Microphotographs of representative Masson's trichrome-stained slides under $\times 40$ magnification. Blue fibers represent collagen, red areas represent muscle. Asterisks show areas where mesh fibers were originally present. Yellow arrow indicates the presence of fatty infiltrates. a Uncoated mesh. b Vitamin E-coated mesh (color figure online)
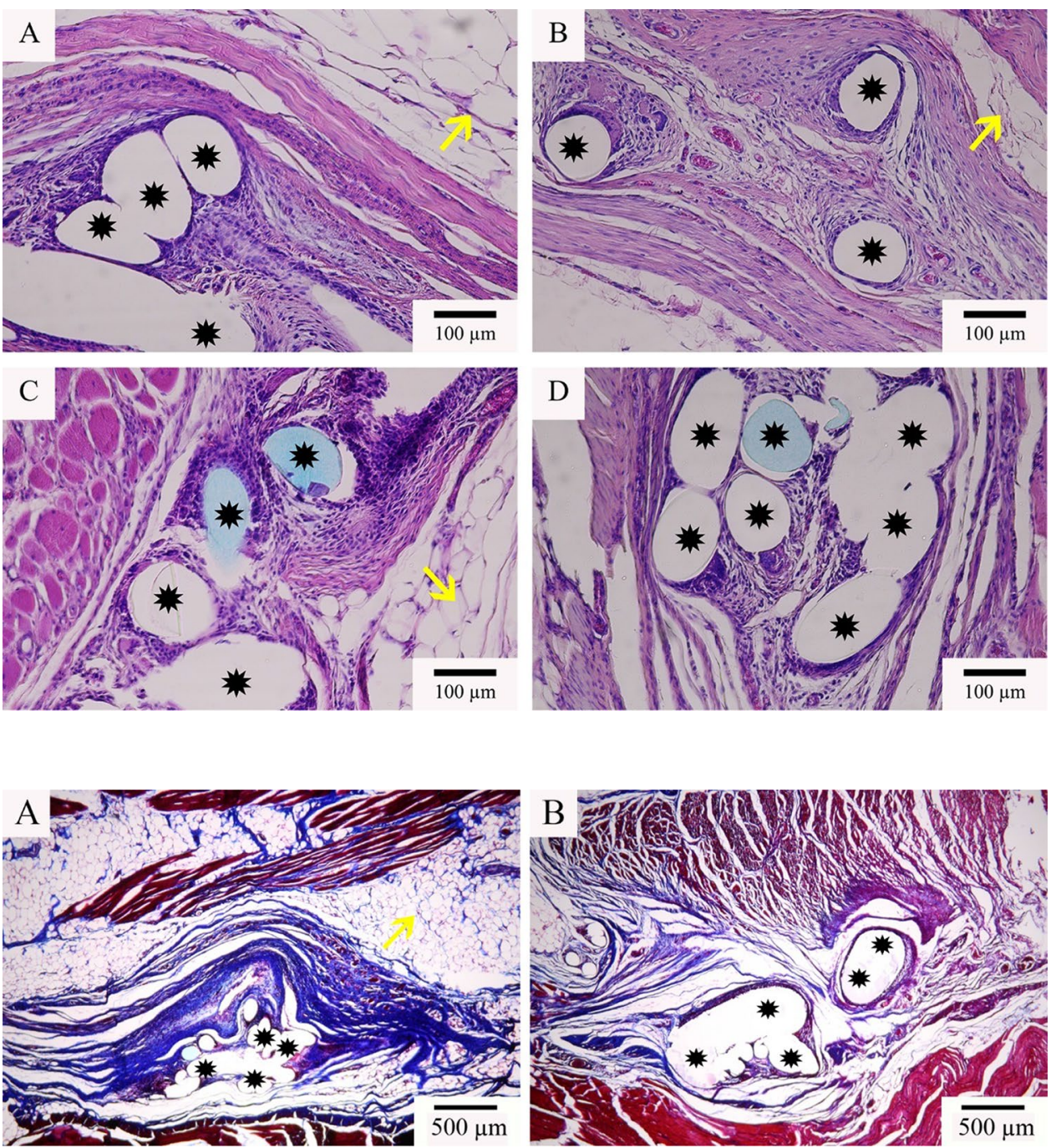
Table 2 Number of macrophages present at meshtissue interface

\begin{tabular}{|c|c|c|c|c|c|c|}
\hline & \multicolumn{4}{|c|}{ Number of macrophages per high powered $(400 \mathrm{x})$ field } & \multirow[t]{3}{*}{$N$} & \multirow[t]{3}{*}{$p$ value } \\
\hline & \multicolumn{2}{|l|}{ Plain } & \multicolumn{2}{|c|}{ Coated } & & \\
\hline & Mean & St.dev & Mean & St.dev & & \\
\hline 1 rabbit & 31 & 5 & 22 & 4 & 6 & 0.023 \\
\hline 2 rabbit & 23 & 4 & 17 & 3 & 6 & 0.015 \\
\hline 3 rabbit & 37 & 4 & 31 & 5 & 6 & 0.045 \\
\hline 4 rabbit & 48 & 5 & 31 & 5 & 6 & 0.0001 \\
\hline 5 rabbit & 28 & 5 & 21 & 3 & 6 & 0.018 \\
\hline $\begin{array}{l}\text { Averaged over } 5 \\
\text { rabbits }\end{array}$ & 32 & 9 & 25 & 6 & 30 & 0.015 \\
\hline
\end{tabular}

vicinity of either of the implants, similarly to the pilot study [20]. The numerical data are presented in Table 2.

Masson's trichrome staining was utilized for characterization of collagen organization, fatty infiltrates, and mesh encapsulation (Fig. 2). Figure 2 shows representative microphotographs of Masson's trichrome-stained slides. Uncoated mesh is encapsulated by a thicker capsule, which is a sign of poor biocompatibility [22]. Thickness of the capsule correlates with collagen organization, which differed significantly. $(p=0.02, n=30$, see Table 3$)$.

Figures 1 and 2 also show significantly higher level of fatty infiltrates in the proximity of the uncoated mesh compared to the coated mesh $(p=0.01, n=30$, see Table 3$)$.
Furthermore, a significantly higher level of neovascularization was observed in the case of the uncoated mesh ( $p=0.015, n=30$, see Table 3 ). Numerical data are given in the Table 3 .

For rabbit \#3 there was no significant difference in number of fatty infiltrates and collagen organization $(p=0.075$ and $p=0.09$, respectively). Additionally, for rabbit \#5 there was no significant difference for the score of fatty infiltrates $(p=0.33)$. All other animals demonstrated significant difference for each parameter individually. Furthermore, the averaged data for five animals show statistically significant difference for all studied parameters.
Table 3 Semi-quantitative histological evaluation of the tissues surrounding uncoated and vitamin E-coated meshes

\begin{tabular}{|c|c|c|c|c|c|c|}
\hline \multirow[t]{3}{*}{ Parameter } & \multicolumn{4}{|c|}{ Histological score } & \multirow[t]{3}{*}{$N$} & \multirow[t]{3}{*}{$p$ value } \\
\hline & \multicolumn{2}{|l|}{ Plain } & \multicolumn{2}{|c|}{ Coated } & & \\
\hline & Mean & St.dev & Mean & St.dev & & \\
\hline \multicolumn{7}{|c|}{ Collagen organization } \\
\hline 1 rabbit & 2.3 & 0.3 & 1.8 & 0.2 & 6 & 0.04 \\
\hline 2 rabbit & 2.6 & 0.3 & 2.1 & 0.1 & 6 & 0.03 \\
\hline 3 rabbit & 2.3 & 0.3 & 2.0 & 0.1 & 6 & 0.09 \\
\hline 4 rabbit & 2.2 & 0.3 & 1.8 & 0.3 & 6 & 0.02 \\
\hline 5 rabbit & 2.6 & 0.6 & 1.9 & 0.3 & 6 & 0.03 \\
\hline Average & 2.3 & 0.3 & 1.9 & 0.2 & 30 & 0.02 \\
\hline \multicolumn{7}{|c|}{ Neo-vascularization } \\
\hline 1 rabbit & 2.1 & 0.5 & 1.7 & 0.3 & 6 & 0.021 \\
\hline 2 rabbit & 2.4 & 0.3 & 1.7 & 0.2 & 6 & 0.031 \\
\hline 3 rabbit & 2.3 & 0.4 & 1.8 & 0.3 & 6 & 0.022 \\
\hline 4 rabbit & 2.0 & 0.3 & 1.7 & 0.2 & 6 & 0.033 \\
\hline 5 rabbit & 2.4 & 0.4 & 1.9 & 0.4 & 6 & 0.021 \\
\hline Average & 2.2 & 0.3 & 1.7 & 0.2 & 30 & 0.015 \\
\hline \multicolumn{7}{|c|}{ Fatty infiltrates } \\
\hline 1 rabbit & 2.3 & 0.3 & 1.6 & 0.2 & 6 & 0.04 \\
\hline 2 rabbit & 2.5 & 0.6 & 1.7 & 0.3 & 6 & 0.047 \\
\hline 3 rabbit & 2.5 & 0.3 & 2 & 0.3 & 6 & 0.075 \\
\hline 4 rabbit & 2.2 & 0.3 & 1.6 & 0.2 & 6 & 0.02 \\
\hline 5 rabbit & 2.5 & 0.5 & 2.1 & 0.4 & 6 & 0.33 \\
\hline Average & 2.4 & 0.3 & 1.8 & 0.3 & 30 & 0.01 \\
\hline
\end{tabular}




\section{Scanning electron microscopy of the mesh surface}

SEM imaging was performed to characterize the surface of the explanted meshes. Figure 3 shows the comparison between the pristine mesh and the pristine mesh that had been soaked in sodium hypochlorite $(10-15 \%$ available chlorine) solution for $12 \mathrm{~h}$ at $50{ }^{\circ} \mathrm{C}$. No difference was observed between the surfaces of the two meshes. This experiment was performed to assess potential effect of the post-explantation cleaning procedure on the structure of the explanted meshes.

Figure 4 shows the surface of the explanted meshes. Vitamin E-coated mesh displayed relatively smooth surface that somewhat resembled pristine mesh (Fig. 4d). There were few areas with small transverse cracks, as seen in the Fig. 4b. Miniscule microcracks were observed mostly at the points of maximum bending: the weave junctions/knits. All the uncoated meshes exhibited substantial level of cracking/crazing in a transverse direction
(Fig. 4c), as well as peeling off at the weave junctions that can be seen in Fig. 4a.

\section{Discussion}

The renowned Austrian surgeon Theodor Billroth who is considered by many as the father of modern abdominal surgery stated in 1878: "If we could artificially produce tissues of the density and toughness of fascia and tendon, the secret of the radical cure of hernia would be discovered" [12]. Decades later Dr. Francis Usher introduced polypropylene mesh implants. Over the years, mesh implantation has become the golden standard in hernia repair, which nowadays is one of the most frequently performed surgeries with over 1 million cases in the U.S. and close to 20 million worldwide each year [1].

Despite the tremendous success of the mesh in reduction of hernia recurrence rate, post-operative complications
Fig. 3 SEM images of the mesh surface. Extrusion lines can be seen in both $\mathbf{a}$ and $\mathbf{b}$. a Pristine mesh, b NaOCl-treated mesh
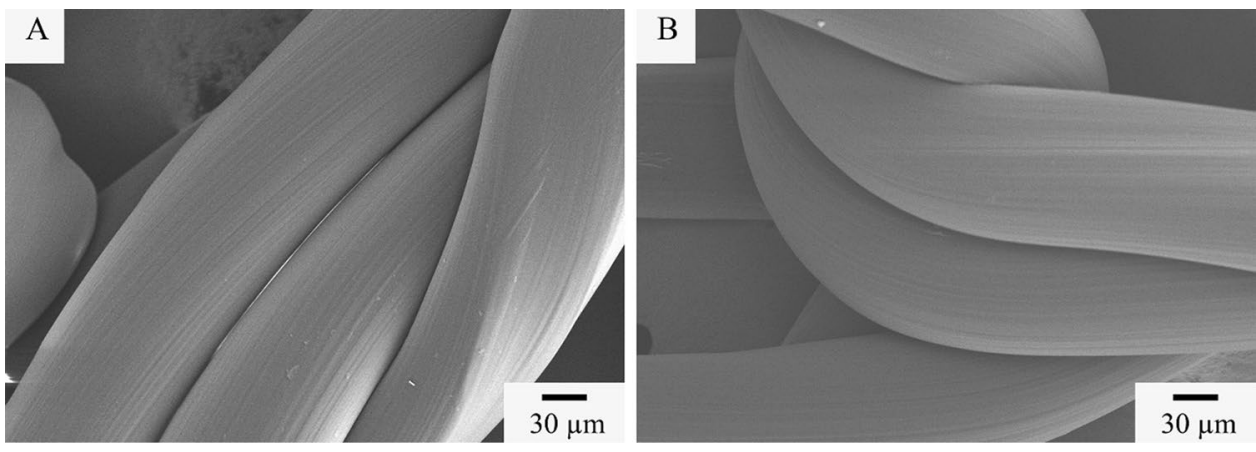

Fig. 4 SEM images of the explanted meshes. Blue arrows indicate peeling off from the surface. Extrusion lines can still be seen, as in the pristine mesh. a, c uncoated mesh $\mathbf{b}, \mathbf{d}$ Vitamin E-coated mesh (color figure online)
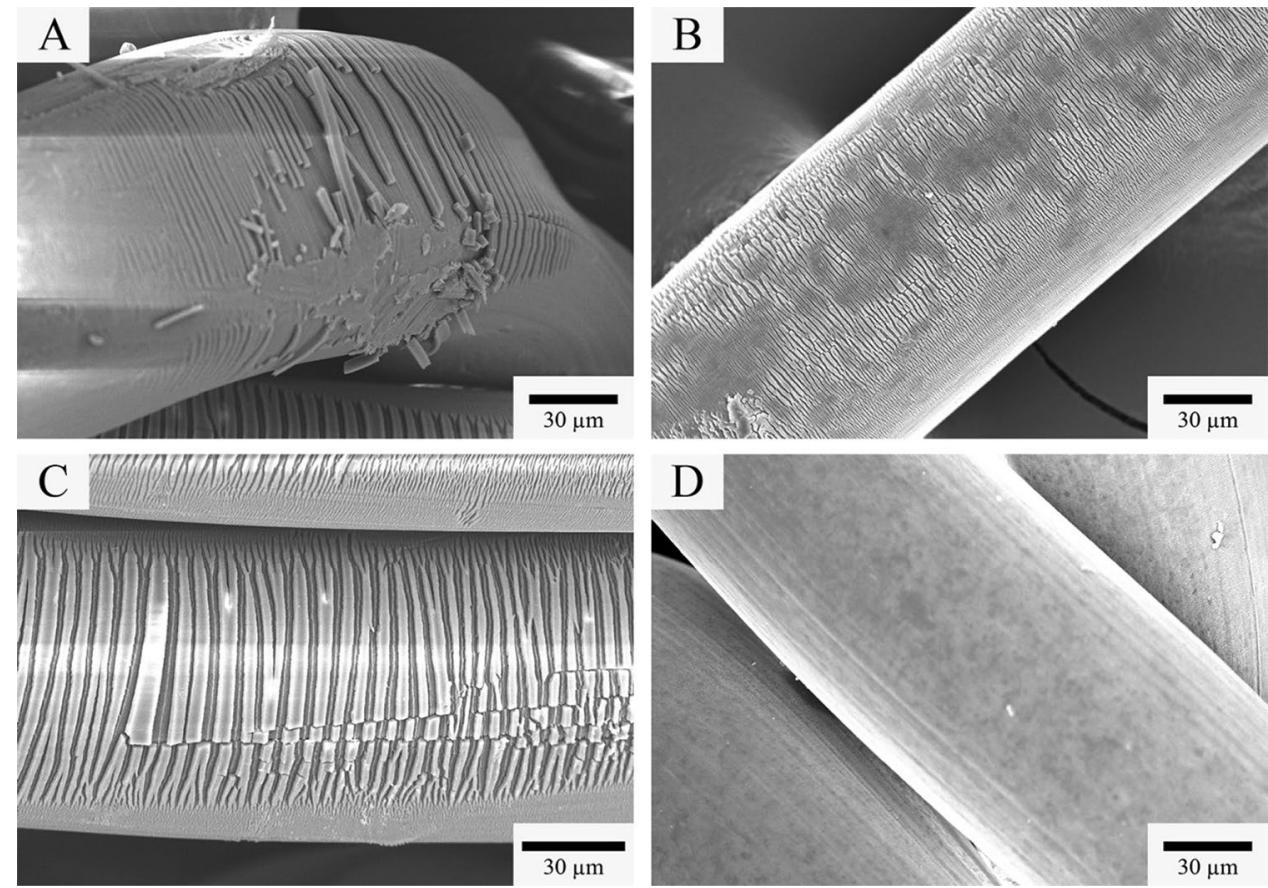
remain a problem. Chronic pain is the most notable one, as nearly a third of the patients experience it [7]. In most cases the only solution is a revision surgery, which puts patient's health through the additional risk and places economic burden on the healthcare system [7].

Oxidative stress appears to be a major factor in the development of chronic pain [23]. The use of antioxidants, therefore, has a potential to alleviate this problem. Vitamin E is a well-known antioxidant that is already used in modification of various implants, such as UHMWPE acetabular liners [18]. Furthermore, vitamin E is hydrophobic, which results in a slow release into an aqueous fluid. We have previously shown that $70 \%$ of bound vitamin $E$ remains on the mesh surface after 10 days of incubation in $10 \%$ bovine serum albumin (BSA) solution, suggesting that the sustained release can be achieved in vivo for during entire wound healing period [20]. All of these considerations make vitamin E a promising bioactive coating that can be applied onto the mesh to reduce inflammatory and oxidative damage to the tissues and the implant.

The implanted mesh and the surrounding tissues were excised on the post-operative day 35. Choice of a 5-week end point allowed for the complete wound healing and provided information on longer-term effects of the coating, which are a major concern for hernioplasty. The most direct measure of the inflammatory response to an implant is the number of macrophages and foreign body giant cells found in the surrounding tissues. Macrophages were observed in the proximity of both vitamin E-coated and uncoated meshes (Fig. 1). These findings are consistent with numerous previous studies in literature [2, 14, 24, 25]. However, the macrophage count near vitamin E-coated meshes was significantly lower compared to uncoated meshes (Table 2). This observation is indicative of reduced inflammatory response in the case of vitamin E-coated meshes. At the same time, no foreign body giant cells were observed in the vicinity of either of the implants, which is consistent with the findings of our pilot study [20].

With respect to the collagen organization, all but one rabbit exhibited significant difference between the uncoated and the coated sample. The collagenous capsule was on the average significantly thicker around the uncoated implants (Fig. 2). According to the previous studies, thick fibrous capsule is associated with an implant shrinkage and increased tissue adhesions [22]. Postsurgical adhesions severely affect quality of life and may cause small-bowel obstruction and difficult revision surgery.

Significant difference in the amount of fat infiltration was observed for three rabbits out of five, and on the average for all five animals (Table 3). Large amount of fat is usually associated with inadequate biocompatibility and elevated connective tissue deposition, which also correlates with increased tissue adhesions [26].
For all rabbits, neovascularization around uncoated meshes occurred to a larger extent than around vitamin E-coated meshes, which is also consistent with the results of our pilot study (Table 3) [20]. It is important to point out that adequate neovascularization is required for the remodeling phase of wound healing, and, therefore, is desirable. However, vast angiogenesis has been associated with poor biocompatibility and intense foreign body reaction [21]. The fact that the uncoated meshes had more newly-formed vessels around them may suggest that either this tissue goes through more extensive remodeling or their wound healing process is delayed compared to the tissue near the vitamin E-coated meshes.

The surface of the explanted meshes was assessed using SEM imaging. Since the removal of tissue from the mesh explants involved treatment by sodium hypochloritea potent oxidant- there was a necessity to ensure that no deterioration was caused by this treatment. Figure 3 shows representative images of the pristine and the hypochloritetreated meshes. No severe deterioration was found on the surface of the treated mesh.

Figure 4 shows SEM images of the explanted meshes. In the case of uncoated meshes, all samples had areas with multiple large cracks oriented perpendicularly to the fiber direction (Fig. 4c). Surface peeling was also detected for some uncoated meshes, particularly at weave junctions (Fig. 4a). The origin of such mesh deterioration has been discussed in a number of in vivo reports [24, 25, 27]. Prevalent opinion is that mesh deterioration occurs in vivo due to the mechanical stress and oxidative degradation $[24,25$, 27]. Alternatively, some authors believe that mesh deterioration occurs after the explantation, during the storage of tissue-coated mesh explants in formaldehyde solution [28]. According to these authors, the observed cracked layer is in fact crosslinked formaldehyde-protein polymer that cannot be removed by conventional hypochlorite cleaning treatment. The first point of view is supported by the fact that all forms of polypropylene are prone to oxidative attack at the tertiary carbon-hydrogen bond [29]. According to Ratner et al., mechanical stresses and environmental conditions act synergistically to cause degradation in polymers, termed by "environmental stress cracking" [30]. As mesh in the abdomen is permanently subjected to the stresses induced by breathing and body movements, it is in a higher energystate than when it is relaxed, and, therefore, is more reactive and susceptible to free radical attack and degradation. Interestingly, Cozad et al. showed that the cracks were concentrated at the weave junctions of the mesh [2]. As these locations have high stress concentrations within the material, they tend to be more reactive than the areas where fibers are relaxed. This elucidates why modifying knit design can improve mesh mechanical performance, such as in the study mentioned in the introduction [11]. 
Thames et al. have argued, however, that the authors of the previous reports failed to consider natural adsorption of proteins, and since initially tissue-mesh explants were kept in formaldehyde solution, the visible cracked layer is in fact a crosslinked formaldehyde-protein polymer [28]. In support of their hypothesis they showed that the cracked layer can be removed using multiple sodium hypochlorite cleaning sequences followed by rigorous vortexing, sonication, and proteinase $\mathrm{K}$ treatment. Their opponents argued that such treatment could have removed the cracked and flaky polymer layer as well, and that the results of their FTIR analysis of the flakes could be explained by the presence of hydroxyl and carbonyl groups due to the oxidation of polypropylene [29].

Our results indicate that cracks were present in both the uncoated and the vitamin E-coated meshes, but there were fewer cracks in the case of the vitamin E-coated meshes and their size was smaller (Fig. 4b), which is indicative of a lower degree of degradation. If the top visible layer was indeed the product of the fixation procedure, which was identical for both the coated and the uncoated meshes, its appearance should have been similar. Thus, our results appear to support the hypothesis of the in vivo origin of the cracks on the mesh fibers.

\section{Conclusion}

In conclusion, results of our animal study indicate that coating polypropylene mesh with vitamin E significantly reduces inflammatory response following hernioplasty and protects the mesh material from oxidative deterioration and environmental stress cracking.

Funding Funding was provided by Clemson University (US) (Grant No. URGC grant).

\section{Compliance with ethical standards}

Conflict of interest No conflict of interest is to be reported for this study.

Ethical approval All applicable international, national and institutional guidelines for the care and use of animals were followed. All animal experimental procedures were conducted according to the protocol approved by the institutional animal care and use committee of Clemson University (AUP-2015-019).

Statement of human rights This article does not contain any studies with human participants performed by any of the authors.

Informed consent This article does not contain any studies with human participants, therefore informed consent was not applicable.

\section{References}

1. Dabbas N, Adams K, Pearson K, Royle G (2011) Frequency of abdominal wall hernias: is classical teaching out of date? JRSM Short Rep. 2:1-6. https://doi.org/10.1258/shorts.2010.010071

2. Cozad MJ, Grant DA, Bachman SL, Grant DN, Ramshaw BJ, Grant SA (2010) Materials characterization of explanted polypropylene, polyethylene terephthalate, and expanded polytetrafluoroethylene composites: Spectral and thermal analysis. J Biomed Mater Res Part B Appl Biomater 94:455-462. https:// doi.org/10.1002/jbm.b.31675

3. Usher F, Ochsner J, Tuttle L (1958) Use of marlex mesh in the repair of incisional hernias. Am Surg 24:969-974

4. Christoffersen MW, Helgstrand F, Rosenberg J, Kehlet H, Bisgaard T (2013) Lower reoperation rate for recurrence after mesh versus sutured elective repair in small umbilical and epigastric hernias. A nationwide register study. World J Surg 37:2548-2552. https://doi.org/10.1007/s00268-013-2160-0

5. Kurtz J, Rael B, Lerma J, Wright C, Khraishi T, Auyang ED (2016) Effects of reactive oxygen species on the physical properties of polypropylene surgical mesh at various concentrations: a model for inflammatory reaction as a cause for mesh embrittlement and failure. Surg Endosc Other Interv Tech 30:3250-3255. https://doi.org/10.1007/s00464-015-4646-3

6. Robinson TN, Clarke JH, Schoen J, Walsh MD (2005) Major mesh-related complications following hernia repair: events reported to the Food and Drug Administration. Surg Endosc Other Interv Tech 19:1556-1560. https://doi.org/10.1007/s0046 4-005-0120-y

7. Kumar S, Wilson RG, Nixon SJ, Macintyre IMC (2002) Chronic pain after laparoscopic and open mesh repair of groin hernia. Br J Surg 89:1476-1479. https://doi.org/10.104 6/j.1365-2168.2002.02260.x

8. Sternschuss G, Ostergard DR, Patel H (2012) Post-implantation alterations of polypropylene in the human. J Urol 188:27-32. https ://doi.org/10.1016/j.juro.2012.02.2559

9. Bryan N, Ahswin H, Smart NJ, Bayon Y, Hunt JA (2012) In vitro activation of human leukocytes in response to contact with synthetic hernia meshes. Clin Biochem 45:672-676. https://doi. org/10.1016/j.clinbiochem.2012.02.026

10. Sánchez O, Rodríguez-Sureda V, Domínguez C, FernándezFigueras T, Vilches A, Llurba E, Alijotas-Reig J (2012) Study of biomaterial-induced macrophage activation, cell-mediated immune response and molecular oxidative damage in patients with dermal bioimplants. Immunobiology 217:44-53. https://doi. org/10.1016/j.imbio.2011.08.003

11. Ibrahim MM, Poveromo LP, Glisson RR, Cornejo A, Farjat AE, Gall K, Levinson H (2018) Modifying hernia mesh design to improve device mechanical performance and promote tensionfree repair. J Biomech 71:43-51. https://doi.org/10.1016/j.jbiom ech.2018.01.022

12. Bilsel Y, Abci I (2012) The search for ideal hernia repair; mesh materials and types. Int J Surg 10:317-321. https://doi. org/10.1016/j.ijsu.2012.05.002

13. Lake SP, Ray S, Zihni AM, Thompson DM, Gluckstein J, Deeken CR (2015) Pore size and pore shape-but not mesh densityalter the mechanical strength of tissue ingrowth and host tissue response to synthetic mesh materials in a porcine model of ventral hernia repair. J Mech Behav Biomed Mater 42:186-197. https:// doi.org/10.1016/j.jmbbm.2014.11.011

14. Emans PJ, Schreinemacher MHF, Gijbels MJJ, Beets GL, Greve JWM, Koole LH, Bouvy ND (2009) Polypropylene meshes to prevent abdominal herniation. Can stable coatings prevent adhesions in the long term? Ann Biomed Eng 37:410-418. https://doi. org/10.1007/s 10439-008-9608-7 
15. Prudente A, Favaro WJ, Reis LO, Riccetto CLZ (2017) Nitric oxide coating polypropylene mesh increases angiogenesis and reduces inflammatory response and apoptosis. Int Urol Nephrol 49:597-605. https://doi.org/10.1007/s11255-017-1520-3

16. Wolf MT, Carruthers CA, Dearth CL, Peter M, Huber A, Burnsed OA, Londono R, Johnson SA, Daly KA, Stahl EC, Freund JM, Medberry CJ, Lisa E, Nieponice A, Amoroso NJ, Badylak SF (2015) HHS Public Access. https://doi.org/10.1002/jbm.a.34671 .Polypropylene

17. Satishkumar R, Sankar S, Yurko Y, Lincourt A, Shipp J, Heniford BT, Vertegel A (2011) Evaluation of the antimicrobial activity of lysostaphin-coated hernia repair meshes. Antimicrob Agents Chemother 55:4379-4385. https://doi.org/10.1128/AAC.01056 $-10$

18. Bracco P, Oral E (2011) Vitamin E-stabilized UHMWPE for total joint implants: a review. Clin Orthop Relat Res 469:2286-2293. https://doi.org/10.1007/s11999-010-1717-6

19. Yetkin G, Uludag M, Citgez B, Karakoc S, Polat N, Kabukcuoglu F (2009) Prevention of peritoneal adhesions by intraperitoneal administration of vitamin $\mathrm{E}$ and human amniotic membrane. Int J Surg. 7:561-565. https://doi.org/10.1016/j.ijsu.2009.09.007

20. Gil D, Rex J, Cobb W, Reukov V, Vertegel A (2018) Antiinflammatory coatings of hernia repair meshes: a pilot study. $\mathrm{J}$ Biomed Mater Res Part B Appl Biomater 106:589-597. https:// doi.org/10.1002/jbm.b.33834

21. Orenstein SB, Saberski ER, Kreutzer DL, Novitsky YW (2012) Comparative analysis of histopathologic effects of synthetic meshes based on material, weight, and pore size in mice. J Surg Res 176:423-429. https://doi.org/10.1016/j.jss.2011.09.031

22. Novitsky YW, Harrell AG, Cristiano JA, Paton BL, Norton HJ, Peindl RD, Kercher KW, Heniford BT (2007) Comparative evaluation of adhesion formation, strength of ingrowth, and textile properties of prosthetic meshes after long-term intra-abdominal implantation in a rabbit. J Surg Res 140:6-11. https://doi. org/10.1016/j.jss.2006.09.015

23. Coda A, Bendavid R, Botto-Micca F, Bossotti M, Bona A (2003) Structural alterations of prosthetic meshes in humans. Hernia 7:29-34. https://doi.org/10.1007/s10029-002-0089-6
24. Clavé A, Yahi H, Hammou JC, Montanari S, Gounon P, Clavé $\mathrm{H}$ (2010) Polypropylene as a reinforcement in pelvic surgery is not inert: comparative analysis of 100 explants. Int Urogynecol J 21:261-270. https://doi.org/10.1007/s00192-009-1021-8

25. Iakovlev VV, Guelcher SA, Bendavid R (2017) Degradation of polypropylene in vivo: a microscopic analysis of meshes explanted from patients. J Biomed Mater Res Part B Appl Biomater 105:237-248. https://doi.org/10.1002/jbm.b.33502

26. Wilkosz S, Epstein J, De Giorgio-Miller A, McLean W, Ireland G, Herrick S (2008) Remodelling of adipose tissue during experimental omental adhesion formation. Br J Surg 95:387-396. https ://doi.org/10.1002/bjs.6073

27. Costello CR, Bachman SL, Grant SA, Cleveland DS, Loy TS, Ramshaw BJ (2007) Characterization of heavyweight and lightweight polypropylene prosthetic mesh explants from a single patient. Surg Innov 14:168-176. https://doi.org/10.1177/15533 50607306356

28. Thames SF, White JB, Ong KL (2017) The myth: in vivo degradation of polypropylene-based meshes. Int Urogynecol J 28:285297. https://doi.org/10.1007/s00192-016-3131-4

29. Thompson M, Guelcher S, Bendavid R, Iakovlev V, Ostergard DR (2017) In vivo polypropylene mesh degradation is hardly a myth. Int Urogynecol J 28:333-335. https://doi.org/10.1007/s0019 2-016-3233-z

30. Ratner BD, Hoffman AS, Schoen FJ, Lemons JE (2012) Biomaterials science: an introduction to materials in medicine, $3 \mathrm{~d}$ edn. Academic Press. https://doi.org/10.1016/B978-0-08-08778 0-8.00148-0

Publisher's Note Springer Nature remains neutral with regard to jurisdictional claims in published maps and institutional affiliations. 